\title{
Factors affecting the level of prolactin in female patients with acute psychotic disorder - Psychiatric hospital "Sveti Ivan"
}

\author{
$\underline{\text { S. Vuk Pisk }}{ }^{1,2}$, K. Matic ${ }^{1}$, E. Ivezic ${ }^{1,2}$, N. Geres ${ }^{1}$, N. Ruljancic ${ }^{1}$, V. Grosic ${ }^{1,2}$, I. Filipcic ${ }^{1,2}$ \\ ${ }^{1}$ Psychiatric Hospital "Sveti Ivan", Zagreb, Croatia \\ ${ }^{2}$ Faculty of Dental Medicine and Health, Josip Juraj Strossmayer University of Osijek
}

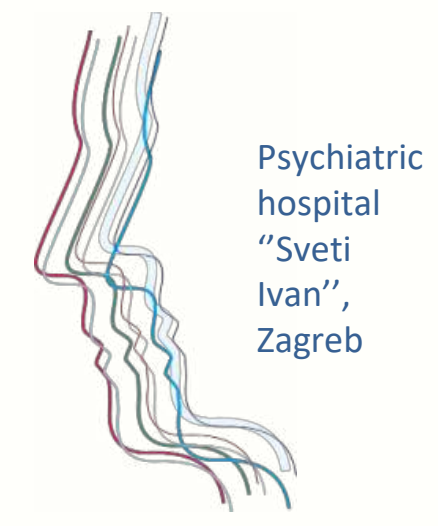

\section{Introduction and objectives}

Previous research suggests a protective effect of prolactin levels in women diagnosed with psychotic disorders: elevated levels of prolactin were associated with milder symptoms. However, the findings have also shown the influence and interdependence of various factors that result in increased levels of prolactin. Thus, the aim of this study was to clarify the role of prolactin in psychotic disorders, i.e. to examine the association of prolactin levels and symptoms severity, as well as to determine the diversity of factors that are correlated with the increase of prolactin concentrations in women with psychotic disorders.

\section{Materials and methods}

A total of 54 adult female psychiatric inpatients diagnosed with psychotic disorders (ICD-10: F20-F29) participated in this study. They completed Positive and Negative Syndrome Scale (PANSS), Depression, Anxiety and Stress Scale (DASS-21) and Glasgow Antipsychotic Side-effect Scale (GASS). Prolactin levels and other laboratory parameters were measured from the blood samples taken in the morning after 30 min rest.

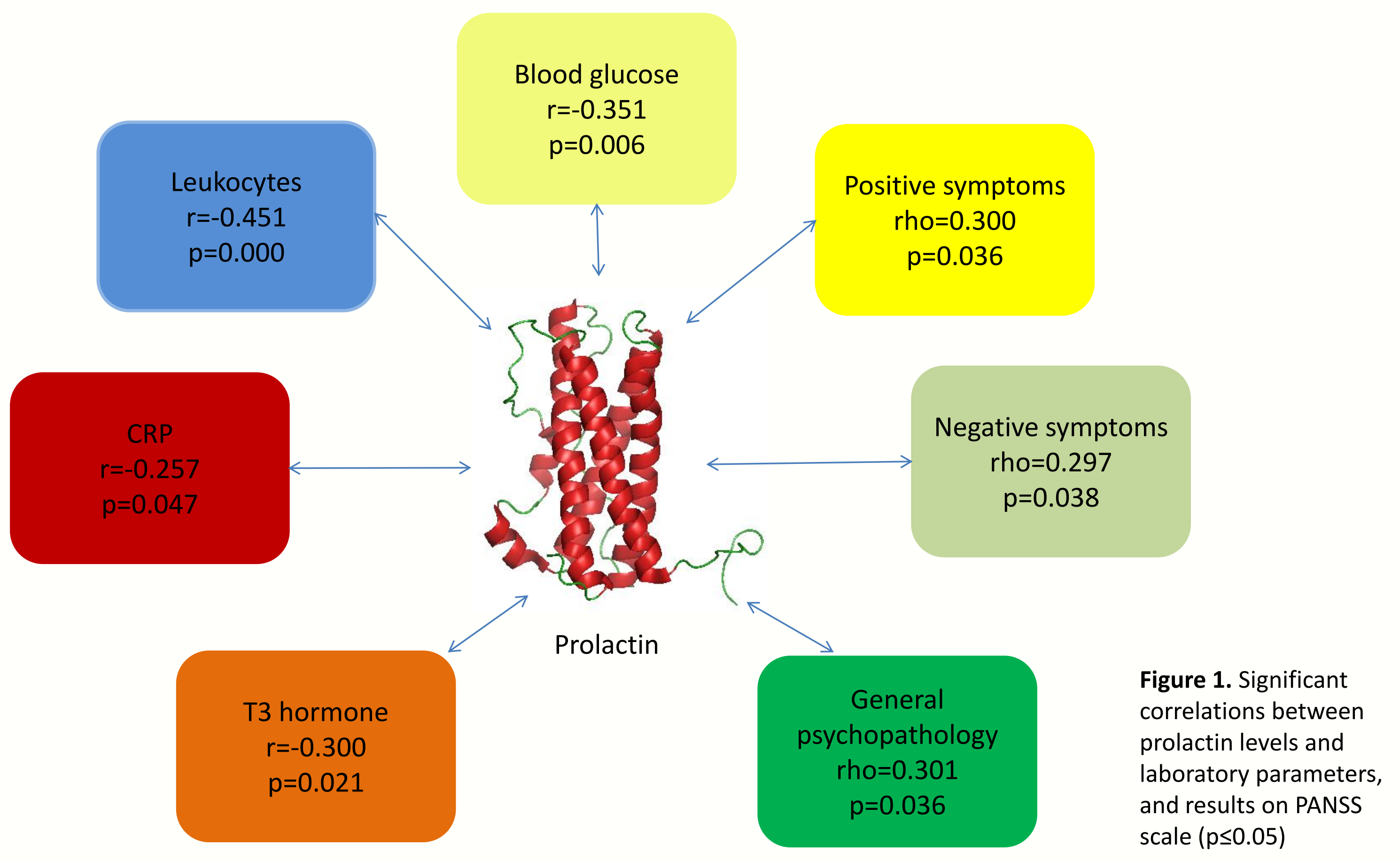

\section{Results}

The findings showed significant correlations between prolactin levels and blood glucose values, leukocytes, CRP and T3 hormone levels: higher prolactin values were associated with lower values of these parameters. On the other hand, elevated prolactin levels positively correlated with symptoms severity according to PANSS scale. No significant correlation was found between concentration of prolactin hormone and results on DASS-21 and GASS scales.

\section{Conclusion}

The results did not confirm the protective effect of prolactin in women: elevated prolatin levels were positively correlated with symptoms severity. The findings also indicated the number of factors that are associated with the elevation in prolactin levels, with a special emphasis on the importance of this knowledge in daily clinical work.

\section{References}

1. Delgado - Alvarado et al. (2018) Plazma prolactin levels are associated with severity of illness in drug-naive firste episode psychosis female patients. Arch. Womans. Menth. Health.

2. Rajkumar RP . (2014) Prolactin and psychopathology in Schizophrenia: Literature Review and reappraisal. Shcizophr. Res. Treatment. 1-12 